\title{
Watershed Based Iris SEgmentation
}

\author{
Maria Frucci ${ }^{1}$, Michele Nappi $^{2}$, and Daniel Riccio ${ }^{3}$ \\ ${ }^{1}$ Istituto di Cibernetica "E. Caianiello", CNR \\ via Campi Flegrei 34, 80078, Napoli \\ ${ }^{2}$ Università degli Studi di Salerno, \\ via Ponte Don Melillo, 84084 Fisciano (Salerno) Italy \\ ${ }^{3}$ Università degli Studi di Napoli Federico II, \\ via Cintia 21, 80126, Napoli \\ m.frucci@cib.na.cnr.it, mnappi@unisa.it, \\ daniel.riccio@unina.it
}

\begin{abstract}
Recently, the research interest on biometric systems and applications has significantly grown up, aiming to bring the benefits of biometrics to the broader range of users. As signal processing and feature extraction play a very important role for biometric applications, they can be thought as a particular subset of pattern recognition techniques. Most of iris biometric systems have been designed for security applications and work on near-infrared (NIR) images. NIR images are not affected by illumination changes in visible light making systems working both in darker and lighter conditions. The reverse of the medal is a very short distance allowed between the acquisition camera and the user, further than a strictly controlled pose of the eye. For those reasons, the viability of NIR image based systems in commercial applications is quite limited. Several efforts have been devoted to designing new iris biometric approaches on color images acquired in visible wavelength light (VW). However, illumination changes significantly affect the iris pattern as well as the periocular region making both segmentation and feature extraction harder than in NIR. In the specific case of iris biometrics, segmentation represents a crucial aspect, as it must be fast as well as accurate. To this aim, a new watershed based approach for iris segmentation in color images is presented in this paper. The watershed transform is exploited to binarize an image of the eye, while circle fitting together with a ranking approach is applied to first approximate the iris boundary with a circle. The experimental results demonstrate this approach to be effective with respect to location accuracy.
\end{abstract}

Keywords: iris segmentation, watershed, circle fitting.

\section{Introduction}

In the last years iris recognition system under constrained acquisition protocols has proven to be effective if compared with automatic fingerprint in several scenarios (e.g., physical access control, computer log-in, international border crossing and national ID cards) [1, 9]. However, the effectiveness and feasibility of iris 
technology under controlled data acquisition conditions may significantly decay in real data scenario with subjects moving and at widely varying distances under both visible wavelength and artificial lighting sources. Although deployed iris recognition systems operate mainly in the near infrared (NIR) spectrum, the majority of ICT devices, including new smart mobile generation, includes visible wavelength cameras. Just the rapid spread of mobile and its evident economic and security implications that it would have in modern societies, is the spring board of throwing for both the detection and recognition of degraded iris images acquired in visible wavelengths. So recently, the scientific community promoted two international evaluation initiatives about iris detection and recognition, named NICE I [13] and NICE II (Noisy Iris Challenge Evaluation) [14]. The UBIRIS database, including heavily degraded iris image, was used as data sources for this event.

First papers on iris detection and recognition, operating in the NIR illumination, have been proposed by Daugman [2] and Wildes [17]. Just a few years after Puhan [11] proposed a novel framework for iris analysis using data source acquired under the visible wavelength light. In this scenario the goal of some participants in NICE I was to optimize the iris localization [15]. Others aimed to reduce the computing time, as for example Li et al [6] that adopt Haar based AdaBoost technique or Labbati and Scotti [5] that propose a novel approach using a rough estimate of the center of the pupil and iris to focus search only in a limited area of the image. Along the same idea ISIS [4] operates only on iris edges extracted by Canny filter performing a precise estimate of the center and radius of the pupil with a low computing time. Iris detection is then determined by using circle fitting algorithm. Even if ISIS demonstrated to be suitable for quasi real-time applications, it always provides, as output, a circle as approximation of both pupil and iris contours.

This paper proposes a novel Watershed based Iris Segmentation, namely WISE that demonstrated to be able in providing the real iris contour and not simply a blurry circle. The watershed transform is a well known that true color and gray level image segmentation performing low asymptotical time complexity of $\mathrm{O}(n)$ and also the real computation speed. WISE, by means of an iterative region growing transform to limit any over-segmentation uses a merging technique specifically designed for the iris context.

The output of the watershed transform suitably thresholded, is subject to a further phase of processing that produces as its final result the contours of both the iris and pupil. The rest of the paper has the following structure. Section 2 describes WISE basic terms, while Sections 3 and 4 show experimental protocol and results. Conclusions and the future work follow in Section 5.

\section{How the WISE Approach Works}

Iris localization (segmentation) is a pattern recognition task aimed to extract the iris texture from a snapshot of the whole periocular region. The most of the existing approaches rely on that iris outer edge is characterized by a strong contrast to the white of the sclera and to darker pixels of the eyelids. Otherwise, the inner edge 
separating the iris from pupil can be more or less contrasted depending on the pigmentation of the iris texture. In both cases, the segmentation process should consider the differences in pixel contrast to accurately locate both iris boundaries. The gradient of an image comes out as a powerful tool to analyze derivatives of brightness, as it underlines the directions of its maximum intensity variations. In particular, gradient information are exploited by the watershed transform [7] to agglomerate pixels in regions, generally named basins and surrounded by gradient banks. As the number of basins tends to be very high, the watershed image is often affected by the problem of over-segmentation; so a merging criterion is implemented to remove week banks and bring together similar regions. In this specific case, the watershed transform is directly applied to an RGB color snapshot of the eye and a binary image is obtained with eyelid and sclera marked as foreground, while iris texture and pupil belonging to the background.

The foreground binary image is inputted to a morphological operator that sets a pixel to 0 if all its 4-connected neighbors are 1, thus only retaining foreground contours. Since the limbus should often be included as part of a more complex contour (see Fig. 2-c), curvature analysis is exploited to split edges at high curvature points. Edges are treated as connected components and circle fitting is applied to find the circle that better approximate the iris boundary. Many approaches search circular shapes, possibly representing the iris, through Hough transform [17] or adaptations [8], which suffer for a high computational cost. Ellipse fitting is faster than the Hough transform, but it also shows some limitations. Indeed, the presence of noise (e.g. spurious branches) may cause the erroneous detection of an elliptical shape even where the expected result would be a circular one. WISE detects circular objects within the image using a precise and fast circle detection procedure presented by Taubin in [16]. The difference between Taubin's approach and ellipse fitting is a relevant aspect to consider; as a matter of fact, given a set of points on a plane, the former identifies a circle which better approximates them, while the latter identifies an ellipse. Since many circles are found while searching for the iris, and WISE does not rely on the Hough transform, a ranking criterion is defined in order to only select the best candidate iris circle.

\subsection{The Watershed}

The watershed is a segmentation approach that builds agglomerations of pixels (region growing) by using gradient ridges as a barrier for growing algorithms [12]. According to this technique, the gradient image is considered as a 3D surface characterized by ridges (banks) and catchment basins (basins). The ridges correspond to curves of maximum gradient, and each basin represents a homogeneous region in which all pixels are connected to a local minimum of the gradient. Generally, the positions of ridges produced by the watershed fits well with the way they would be placed by the human perception. Unfortunately, the number of basins may be very high, as it is proportional to the number of local minima of the gradient, thus resulting in an over-segmented image. In such cases, specific merging criteria are designed to bring together basins with very similar characteristics. 
WISE exploits the watershed to transform an RGB image into a binary one, in which iris is separated as much as possible from the rest of the eye elements (sclera, eyelid, eyelashes). First of all, a median filter with window size $n \times n$ ( $n$ has been set to 7 , according to the resolution of the input images $800 \times 600$ pixels) is applied to the image (Fig. 1-b), in order to remove noise as well as to smooth sharper color differences (a first way of preventing over-segmentation). In order to produce the initial partitioning of the image, the Sobel's filter is used to extract the gradient information that is inputted to the watershed transform (Fig. 1-c). Regions $D_{i}$ produced by the watershed are a covering of the image (Fig. 1-d), that is they are all disjoint $\left(D_{i} \cap D_{j}=\varnothing\right)$, while their union gives back exactly the whole image $\left(\cup D_{i}=I\right)$. In order to reduce the number of segmented regions, a color based merging criterion is implemented. In more details, each region $D_{i}$ is assigned with its average color $C_{i}=\left(R_{i}, G_{i}, B_{i}\right)$ that is computed as the mean value of the colors of pixels belonging to it. The distance between average colors $C_{i}$ and $C_{j}$ of each pair of adjacent regions is computed according to the following formula (eq. 1):

$$
d\left(C_{i}, C_{j}\right)=\sqrt{\left(R_{i}-R_{j}\right)^{2}+\left(G_{i}-G_{j}\right)^{2}+\left(B_{i}-B_{j}\right)^{2}},
$$

The color distance $d$ represents the criterion WISE uses to decide if two adjacent basins must be merged into a larger region. In other words, given a basin $D_{i}$, the color distances to its adjacent basins are calculated and it is merged with the one having the minimum color distance, provided that this distance is lower than a fixed threshold $\delta_{\mathrm{d}}$ (in this work $\delta_{\mathrm{d}}$ has been experimentally set to 50) (Fig. 1-e). The average color is then re-computed over all pixels belonging to the new basin (Fig. 1-f). Assuming that white color (W) is represented by R-,G- and B-component with value 255 , while black (K) has R-, G- and B-component equal to 0 , the couple of distances $d\left(C_{i}, W\right)$ and $d\left(C_{i}, K\right)$ is computed for each region $D_{i}$. The mean values $W_{C}$ and $K_{C}$ are obtained by averaging $d\left(C_{i}, W\right)$ (symmetrically $d\left(C_{i}, K\right)$ ) distances over all regions $D_{i}$. Furthermore, the distance $d(W, K)$ is considered as the maximum value the distance $d$ can assume and it equals to 441.6730. In order to separate foreground regions from the background, that is to produce a binarization of the input image, the value of the distance $d$ must be thresholded. In other words, we need a threshold $\varepsilon_{d}$ to which color distances must be compared with. Distances $d_{W}$ and $d_{K}$ are defined as follows (eq. 2):

$$
\begin{aligned}
& d_{W}=W_{C} \cdot\left(\frac{W_{C}}{d(W, K)}+\frac{1}{2}\right), \\
& d_{K}=K_{C} \cdot\left(\frac{K_{C}}{d(W, K)}+\frac{1}{2}\right),
\end{aligned}
$$

The threshold $\varepsilon_{d}$ is used to binarize all regions produced by the watershed and is computed just once for a given image according to the following equation (eq. 3 ): 


$$
\varepsilon_{d}=d(W, K) \cdot \frac{d_{K}}{d_{W}+d_{K}},
$$

Obviously it changes from an image to another, as it depends on distances between pair of regions that come out from the watershed (Fig. 1-g). Notice that in the binary image (Fig. 1-g) the foreground corresponds to black regions, while the background is represented by the white ones. The separation between foreground and background in the binary image is further improved by applying a refining process that can switch the membership of a basin from foreground into background. In more details, let be $C_{F}$ the average color of the foreground computed by averaging all $C_{i}$ of the basins $D_{i}$ that belong to it. In a symmetric way, $C_{B}$ is calculated as the average color of the background. For each basin $D_{i}$ in foreground the distance of the corresponding $C_{i}$ to the average colors $C_{F}$ and $C_{B}$ is calculated; so if $D_{i}$ belongs to the foreground, while holding that $\mathrm{d}\left(\mathrm{C}_{\mathrm{i}}, \mathrm{C}_{\mathrm{B}}\right) \leq \mathrm{d}\left(\mathrm{C}_{\mathrm{i}}, \mathrm{C}_{\mathrm{F}}\right)$, its membership is switched into background (see Fig. 1-h).
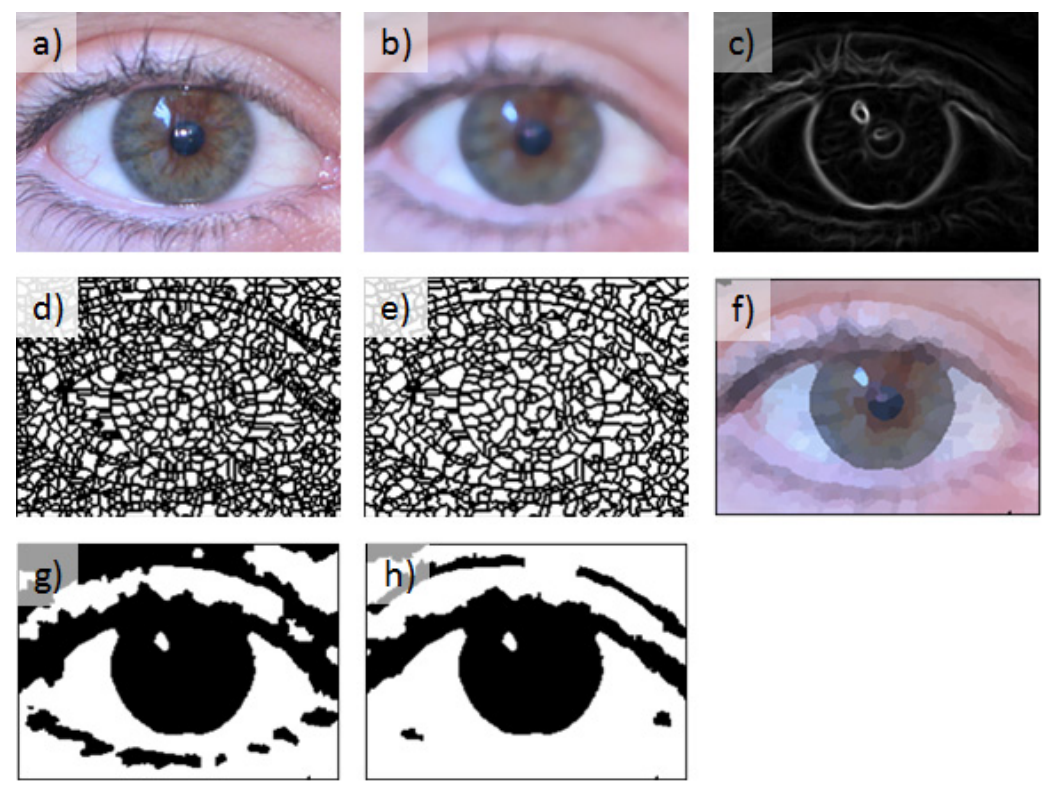

Fig. 1. Some images produced by WISE during the binarization process: a) the original colour image, b) the smoothed image, c) the gradient image, d) the output of the watershed transform (1100 basins), e) the pool of basins after the merging operation (690 basins), f) the quantized image, g) the binary image produced by thresholding the watershed, h) the improved binary image

\subsection{Curvature Analysis and Circle Fitting}

The output of the thresholded watershed transform is a binary image, in which the background is represented by black pixels and the foreground by white ones. As 
WISE searches for a circle approximating the iris boundary, a considerably amount of computation can be saved by focusing the attention only on contour information. To this aim, an edge image is computed by applying a morphological operator to the binary mask produced by the watershed (Fig. 2-c). This operator sets a pixel to 0 if all its 4-connected neighbors are 1 , thus leaving only the boundary pixels on. It is worth noting that the watershed transform well separate lighter regions from darker ones in the original image. For this reason the sclera is marked as foreground in the binary mask, unlike the iris texture which belongs to the background. Pupil and iris correspond to a black concavity hollowed into the foreground region of the sclera, making the iris boundary to be part of the sclera contour. It is essential to isolate pixels belonging to the iris boundary from the rest of the sclera contour, in such a way that a circle fitting technique can be used to approximate it. Fig. 2-c shows that contour direction changes very smoothly along the iris boundary, while a sharp bend in this line characterizes its end points. This peculiar behavior of the iris contour makes the curvature analysis particularly suitable for detecting sharp diverts in the edge image.

However, the aim of this processing step is only to cut edges at points with high curvature and the point-wise computation of the curvature of a generic shape entails a high computational cost, so we can rather estimate it by using such an heuristic criterion that would be easier to calculate. Edges are treated as connected components. For each connected component $C$ a contour tracing algorithm is applied to sorts all points $P_{k}=\left(x_{k}, y_{k}\right)$ belonging to $C$ in a list $L_{C}=\left\{\mathrm{P}_{l}, P_{2}, \ldots, \mathrm{P}_{n}\right\}$. According to this list, the contour $C$ is linearly scanned point by point. For each $P_{k}$, a further point $P_{k+t}$ is also considered, where $\mathrm{t}=4 \cdot\left\lfloor\log _{2}(|C|)\right\rfloor$ is a parameter, whose value is proportional to the contour length. In particular, WISE considers the midpoint $M=\left(m_{x}, m_{y}\right)$ of the segment $S$ ended by $P_{k}$ and $P_{k+t}$ and calculates the Euclidean distance $d_{k, t}\left(M, P_{n}\right)$ between $M$ and $P_{n}$, where $n=\lfloor(k+t) / 2\rfloor$. The values of $d_{k, t}\left(M, P_{n}\right)$ are divided by $d_{\max }=\underset{k}{\arg \max }\left(d_{k, t}\right)$, to ensure they belong to the range [0,1]. The higher is $d_{k, t}\left(M, P_{n}\right)$, the larger is the value of the estimated curvature at point $P_{k}$. Given a fixed threshold $\sigma$ (in this case $\sigma=0.5$ ), all points $P_{k}$ for which the estimated curvature $d_{k, t}$ is larger than $\sigma$ are deleted from $L_{C}$, so that a complex contour $C$ is partitioned in a set of smoothed edges $E_{1}, E_{2}, \ldots, E_{q}$, as shown in Fig. 2-c.

A connected component search is applied to the resulting edge image, so that all pixels belonging to the edge $E_{i}$ are grouped in the same component $Q_{i}$ (Fig. 2-d), while only those containing a number of pixels greater than a threshold $T h_{Q}$ are included in a unique list $H$ (here $T h_{Q}=150$ ). Taubin's algorithm [16] is applied to each element $Q_{i}$ in $H$ to compute the corresponding circle $R_{i}$ and its approximation error $e\left(Q_{i}, R_{i}\right) \in[0,1]$. The final list $H_{Q}$ is obtained by pruning from $H$ all components whose circles are not completely contained inside the image. In order to identify the iris, each circle $R_{i}$ undergoes a voting procedure, according to a score value $s_{i}$, which is computed as follows: 


$$
\begin{gathered}
\qquad s_{i}=e_{h}-e\left(Q_{i}, R_{i}\right), \\
\text { where } h=\underset{i}{\arg \max }\left(e\left(Q_{i}, R_{i}\right)\right)
\end{gathered}
$$

The circle $R_{\max }$ with highest score $s_{\max }$ is considered as the circular shape which better approximates the iris (Fig. 2-e).
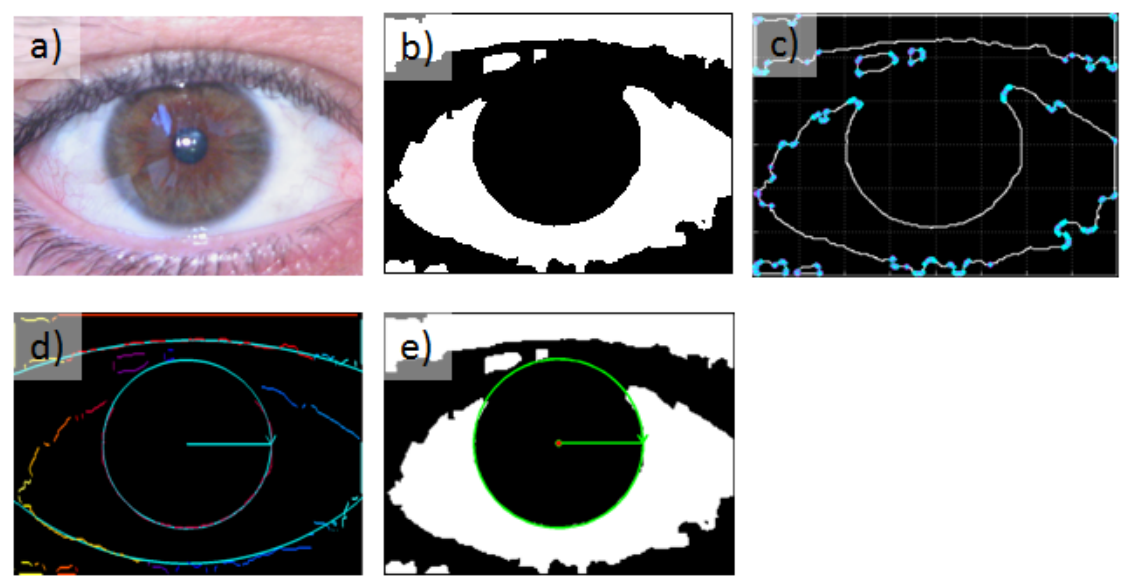

Fig. 2. A graphical representation of outputs produced by the intermediate steps of WISE: a) the original colour image, b) the binary image produced by the thresholded watershed, c) points where curvature is larger than a given threshold $\sigma$, d) circle fitting of the connected components, e) the circle with best rank

\section{The Experimental Setup and Results}

We evaluated the precision of iris detection and compared the results obtained by our WISE system with those provided by a system built from Masek's implementation of various iris-related algorithms, where segmentation is performed according to Wildes' method [17] as well as those reached by the IS $_{\text {IS }}$ approach [4].

In order to assess the accuracy of the iris segmentation approaches we considered in this paper, a precise manual localization of the iris centre and radius was used as ground truth for each iris image. A manual annotating system was used, in which iris images were shown to a human operator who selected their centre $(x, y)$ and radius $(r)$ by clicking them with the mouse.

The different approaches were tested on UBIRIS v1 Session 2 database [10]. The UBIRIS v1s2 database is composed of 1877 images with a resolution of $800 \times 600$ pixels, which have been collected from 241 persons. The presented experimental results report location accuracy iris centre $(x, y)$ and radius $(r)$. Table 1 reports the relative precision of iris location by ISIS, Wildes, and WISE with respect to manual segmentation. Mean and variance of error are relative to the image size. Image coordinates are normalized according to the respective direction (x with 800, $\mathrm{y}$ with 600). Radiuses are normalized according to the horizontal direction. Experimental 
results demonstrated that if the distances are greater than a 20 pixels threshold, the iris can't be detected because the eye is closed or the detection is wrong. The columns "no out." ("no outliers") report mean and variance computed by eliminating completely wrong locations (error greater than 20 pixels) or also UBIRIS closed eyes. Under the method's label we report the number of eliminated outliers.

Table 1. Location results for UBIRIS database

\begin{tabular}{|c|c|c|c|c|c|c|c|}
\hline \multirow{2}{*}{\multicolumn{2}{|c|}{ UBIRIS v1s2 }} & \multicolumn{2}{|c|}{ Iris $x$} & \multicolumn{2}{|c|}{ Iris y } & \multicolumn{2}{|c|}{ Iris radius } \\
\hline & & all & no out. & all & no out. & all & no out. \\
\hline ISIS & mean & 2.4 & 0.3 & 4.3 & 1.6 & 2.2 & 0.8 \\
\hline out. $=12 \%$ & std & 6.3 & 0.3 & 6.4 & 1.4 & 5.0 & 0.6 \\
\hline WILDES & mean & 24.8 & 24.9 & 24.8 & 24.9 & 10.4 & 10.2 \\
\hline out. $=38 \%$ & std & 7.0 & 2.4 & 7.9 & 3.7 & 1.7 & 1.7 \\
\hline WISE & mean & 1.3 & 0.5 & 2.2 & 1.7 & 1.2 & 0.8 \\
\hline out. $=18 \%$ & std & 4.2 & 0.8 & 3.7 & 1.9 & 2.3 & 1.1 \\
\hline
\end{tabular}

A first observation is the slightly worst performances on the vertical coordinate of the pupil centre. This might be due to eyelids, which especially affect such dimension. When outliers are not dropped from whole set of iris images, WISE always outperforms the other methods. Otherwise, in the case in which outliers are not considered for evaluating the segmentation accuracy, WISE performance overcomes that of WILDES, being comparable with that of ISIS. Similarly, the number of outliers produced by WISE is comparable to that provided by ISIS, which is the smallest one.

It is worth to notice that even when images are affected by a wide number of problems due to occlusions or reflections, WISE still achieves acceptable performances, while those by the other methods dramatically decrease.

\section{Conclusions}

This paper has illustrated a new watershed based approach for iris segmentation on color images acquired in visible wavelength light. The technique has been designed to provide a more robust segmentation that is a crucial step when illumination changes significantly. The adopted watershed transform combines a classical iterative region growing and a novel merging technique specifically designed for the iris context. The experimental results demonstrate this approach to be effective with respect to location accuracy if compared with today's algorithms.

\section{References}

1. Daugman, J.G.: New methods in iris recognition. IEEE Transactions on Systems, Man, and Cybernetics - Part B: Cybernetics 37(5), 1167-1175 (2007)

2. Daugman, J.G.: How iris recognition works. IEEE Transactions on Circuits and Systems for Video Technology 14(1), 21-30 (2004) 
3. Daugman, J.G.: How Iris Recognition Works. IEEE Trans. on CSVT 14(1), 21-30 (2004)

4. De Marsico, M., Nappi, M., Riccio, D.: IS_IS: Iris Segmentation for Identification Systems. In: Proc. of the International Conference on Pattern Recognition, pp. 2857-2860 (2010)

5. Donida Labati, R., Scotti, F.: Noisy iris segmentation with boundary regularization and reflections removal. Image and Vision Computing 28(2), 270-277 (2010)

6. Li, P., Liu, X., Xiao, L., Song, Q.: Robust and accurate iris segmentation in very noisy iris images. Image and Vision Computing 28(2), 246-253 (2010)

7. Meyer, F.: Color image segmentation. In: Proc. of the International Conference on Image Processing and its Applications, pp. 303-306 (1992)

8. Nguyen, V.H., Hakil, K.: A Novel Circle Detection Method for Iris Segmentation. In: Proc. of the Congress on Image and Signal Processing, vol. 3, pp. 620-624 (2008)

9. Phillips, P.J., Scruggs, T., O’Toole, A., Flynn, P.J., Bowyer, K.W., Schott, C., Sharpe, M.: FRVT 2006 and ICE 2006 Large-Scale (2006)

10. Proenca, H., Alexandre, L.A.: UBIRIS: A noisy iris image database. In: Proc. of the International Conference on Image Analysis and Processing, pp. 970-977 (2005)

11. Puhan, N.B., Sudha, N.: A novel iris database indexing method using the iris color. In: Proc. of the 3rd IEEE Conf. on Industrial Electronics and Applications, pp. 1886-1891 (2008)

12. Roerdink, J.B.T.M., Meijster, A.: The watershed transform: definitions, algorithms and parallelization strategies. Fundamenta Informaticae 41, 187-228 (2001)

13. Special Issue on the Segmentation of Visible Wavelength Iris Images Captured At-adistance and On-the-move. Image and Vision Computing 28 (2010)

14. Special Issue on the Recognition of Visible Wavelength Iris Images Captured At-adistance and On-the-move. Pattern Recognition Letters 33 (2012)

15. Tan, T., He, Z., Sun, Z.: Efficient and robust segmentation of noisy iris images for noncooperative iris recognition. Image and Vision Computing 28(2), 223-230 (2010)

16. Taubin, G.: Estimation of Planar Curves, Surfaces And Nonplanar Space Curves Defined By Implicit Equations, With Applications To Edge And Range Image Segmentation. IEEE Trans. on PAMI 13, 1115-1138 (1991)

17. Wildes, R.: Iris recognition: an emerging biometric technology. Proceedings of the IEEE 85(9), 1348-1363 (1997) 